\title{
British Thoracic Society guideline for non-CF bronchiectasis
}

\section{C Pasteur, ${ }^{1}$ D Bilton, ${ }^{2}$ A T Hill, ${ }^{3}$ on behalf of the British Thoracic Society Non-CF Bronchiectasis Guideline Group}

The BTS non-cystic fibrosis (CF) bronchiectasis guideline ${ }^{1}$ is published as a supplement to this issue of the journal. It is a summary of the evidence to support the investigation and management of both children and adults with bronchiectasis. The guideline has been written with the help of multidisciplinary working groups that included adult and paediatric respiratory physicians, microbiologists, physiotherapists, specialist nurses, a general practitioner, immunologist, radiologist, thoracic surgeon and a patient with bronchiectasis.

The guideline has been divided into sections that relate to all aspects of investigation and management. Headings are often in the form of a question that the authors hope will relate to day-to-day clinical practice. Sections 2 and 3 highlight the importance of, first, looking for an underlying cause in patients with bronchiectasis and, second, who and how to investigate such patients. Integration of services with other clinical teams such as radiology and microbiology is essential. Some patients may require referral to other units, for instance for the investigation of ciliary defects or immune deficiency, and pathways for organising this are suggested. Section 4 reviews the

${ }^{1}$ Norfolk and Norwich University Hospital, Norwich, UK; ${ }^{2}$ Royal Brompton Hospital, London, UK; ${ }^{3}$ Royal Infirmary of Edinburgh, Edinburgh, UK evidence for the differing roles of primary and secondary care, specialist nursing and the multidisciplinary team. Not all patients will require secondary care follow-up, and designing integrated pathways is a significant challenge for the future. It also focuses on non-antibiotic therapies such as airway clearance and airway pharmacotherapy. The use of antibiotics is discussed in Section 5. The guideline finishes with Section 6, where the role of surgery and non-invasive ventilation is discussed. Lung resection surgery is performed much less frequently now, but there is still a role in carefully selected cases.

One of the biggest challenges for the guideline has been formulating the recommendations in Section 5 for antibiotic therapy (particularly for the use of antibiotics in children with bronchiectasis where there is very little direct evidence) and the majority are grade $\mathrm{D}$. The Guideline Group felt it was important not simply to extrapolate evidence from clinical practice in CF and studies solely relating to $\mathrm{CF}$ were not included in the evidence base. There are a perhaps surprising number of studies looking at the use of antibiotics in adults, extending back to work done in the 1950s and 1960s. While the methodology may be criticised and some of the antibiotics studied are no longer in use, we have been able to use these studies to inform the recommendations and hope that this makes a secure basis for the design of trials in the future.

The use of long-term prophylactic antibiotics is one area where there is an opportunity for informative clinical studies and much of what is common clinical practice is not based on a strong evidence base. A word of caution is perhaps warranted with regard to the use of long-term macrolides in patients with non-CF bronchiectasis colonised with Pseudomonas aeruginosa. The improved outcomes using long-term macrolides in CF now come with concerns about the possible increased incidence of non-tuberculous mycobacteria together with strains of mycobacteria that are then resistant to macrolides. CF and non-CF bronchiectasis are not the same disease in terms of prognosis, and further studies to address both the efficacy and long-term safety are crucial before this approach is adopted widely.

There are many challenges ahead for the management of patients with bronchiectasis, in particular the integration of delivery between primary and secondary care, safe and cost-effective use of antibiotics and other resources and directing patients in becoming involved in their own care, where far less work has been done than in other respiratory disorders such as asthma and chronic obstructive pulmonary disease. The best advocates for high quality care will be the continued interest and determination of the health professionals working in the field and the patients themselves.

Competing interests None.

Provenance and peer review Commissioned; not externally peer reviewed.

Thorax 2010;65:577.

doi:10.1136/thx.2010.142778

\section{REFERENCE}

1. Pasteur MC, Bilton D, Hill AT. British Thoracic Society guideline for non-CF bronchiectasis. Thorax 2010;65(Suppl I):i1-i58 